\title{
Impact of pointing error on SISO/MISO drones swarm-based free space optical system in weak turbulence regime
}

\author{
Abdullah Jameel Mahdi ${ }^{1}$, Wamidh Jalil Mazher ${ }^{2}$, Osman Nuri Ucan ${ }^{3}$ \\ ${ }^{1}$ Collage of Engineering (Electromechanical Engineering Department), University of Samarra, Iraq \\ ${ }^{2}$ Thi Qar Technical College (Electrical Technical Department), Southern Technical University, Iraq \\ ${ }^{3}$ The Graduate School of Science and Engineering, Altinbas University, Turkey
}

\section{Article Info}

Article history:

Received Feb 25, 2021

Revised Jul 13, 2021

Accepted Jul 18, 2021

\section{Keywords:}

Average bit error rate

Free space optical

Multiple input single output

Pointing error angle

Signal-to-noise ratio

Single input single output

\begin{abstract}
Applying the drone-based free space optical (FSO) technology is recent in communication systems. The FSO technology has high-security features due to narrow beamwidth, insusceptible to interferences, free license, and landline connection is not appropriate. However, these advantages face many obstacles that affect the system's performance, such as random weather conditions and misalignment. The pointing error $H_{p}$ is one of the critical factors of the channel gain $H$. The related parameters of the $H_{p}$ factor: the pointing error angles $\theta_{r}$ and the path length $Z$, were manipulated to extract the applicable values at various receiver diameter values. The proposed system has two topologies: single input single output (SISO) and multiple input single output (MISO), flying in weak atmospheric turbulence. The simulation was done using MATLAB software 2020. The average bit error rate (ABER) for the system versus signal-to-noise ratio (SNR) were verified and analyzed. The results showed that at $\theta_{r}=10^{-3} \mathrm{rad}, \mathrm{Z}$ increased in the range $10 \sim 100 \mathrm{~m}$ for each one-centimeter increase of $D_{R}$. At $\theta_{r}=10^{-2} \mathrm{rad}$, the applicable $\mathrm{Z}$ was nearly $10 \%$ of the link distance $\mathrm{Z}$ when $\theta_{r}=10^{-3} \mathrm{rad}$ was applied. Consequently, an increase in $\theta_{r}$ must correspond decrease in $\mathrm{Z}$ and vice versa to maintain the system at high performance.
\end{abstract}

This is an open access article under the CC BY-SA license.

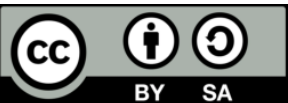

\section{Corresponding Author:}

Abdullah Jameel Mahdi

Electromechanical Engineering Department

University of Samarra

Saladin, Samarra, Iraq

Email: Abdullah.j.m@uosamarra.edu.iq

\section{INTRODUCTION}

Unmanned aerial vehicles (UAVs) are a new technology recently adopted in varying civil and military applications such as traffic jam monitoring, backup communication link in the disaster area, and oversight behind the enemy line. The drone is one type of UAV that is used in low altitudes and short distances. The drone's communication link is unstable due to channel mobility. The major problem from a non-stationary link is the optical beam pointing error (misalignment) between the transmitter and the receiver. Additional factors that help increase the pointing error are the turbulence-induced beam wander, the vibration of the transceivers' platforms, thermal expansion, building sway, and earthquake for the free space optical (FSO) link. The pointing error $H_{p}$ factor, the atmospheric attenuation $H_{a}$, and the $H_{f}$ composed the channel gain $H$. This paper focused on the $H_{p}$ factor's related parameters by manipulating the pointing error angle $\theta_{r}$ and the link distance $Z$. The goal is to extract the permitted pointing error angles $\theta_{r}$ and the link distances $\mathrm{Z}$ at different receiver diameter $\mathrm{D}_{\mathrm{R}}$ in order to keep the system at high performance. 
Most researchers studied the system performance taken the pointing error in the account using fixed terrestrial optical link such as the researches in [1]. The system model in [2] had consisted of multi-hop FSO communication to determine the outage probability when the system was impaired by weak turbulence and misalignment. The designed FSO system in [3] had multiple transceivers of four transmitters \& receivers. It analyzed the Q-factor, bit-error rate (BER), beam divergence, and received power with different climate conditions of clear air, haze, moderate to light fog at wavelengths $850 \mathrm{~nm}$ and $1550 \mathrm{~nm}$. The previous studies on the UAVs-based FSO communication link are relatively recent and rare, especially the effect of pointing error such as [4], which considered the atmospheric attenuation $H_{a}$ and atmospheric turbulence effect $\mathrm{H}_{\mathrm{f}}$ factors without taking the pointing error $H_{p}$ factor into account. Fawaz et al. [4], the authors studied the UAV system's improvement by using relay-assisted FSO to ameliorate the effects of the various atmospheric impairments on the quality of the FSO signal. The review in [5] investigated the ground-UAV and UAV-UAV links scenarios and the possible inter-UAV links scenario in the presence of the impact of atmospheric turbulence on performance. The target BER had been achieved by optimum selecting the beamwidth to decrease the transmitted power, as shown in [6]. The survey done in [7] highlighted the continuous movement and changing relative speeds of participating members, making sustaining a line-ofsight (LOS) FSO link in the UAV swarm scenario difficult. The research [8] measured the performance of a non-static and slanted link between the fixed station at the ground and the UAV. A more recent study [9] aimed to derive the optimal 3D coordinates of UAV relay and optimal optical beam pattern to minimize the outage probability by characterizing source-to-relay and relay-to-destination channel models.

\section{RESEARCH METHOD}

Various UAV communication architectures network can be formed as explained in [9], [10]. The proposed system configuration is as shown in Figure 1. It composed of single input single output (SISO) and multiple input single output (MISO) channels, where the link between the drones in an arm and the connection between the ground station (GS) and the main drone $\left(\mathrm{D}_{\mathrm{M}}\right)$ is the SISO channel, and the link connecting the last drones in both arms $\left(\mathrm{D}_{\mathrm{aN1}}\right.$ and $\left.\mathrm{D}_{\mathrm{aM} 1}\right)$ and the $\mathrm{D}_{\mathrm{M}}$ is the MISO channel. The proposed system applied in detecting and making deep decisions for area surveillance (e.g., oil pipeline leak) [11], which aimed to calculate the number of drones in the system that covered a specified area and examined the effect of increasing the number of drones on the system performance, but it did not consider the pointing error $\mathrm{H}_{\mathrm{p}}$ factor. The SISO topology has one transmitting aperture at the transmitter end and one receiving aperture at the receiver end. The MISO has multiple transmitter antennas where the optical signals have been sent. Only one receiving antenna receives the optical signals from multiple transmitting antennas, which means various sources are available and only one available destination [12]. Twice of the SISO topology in the proposed system, the first between the drones in both arms can be considered a horizontal link. The second is the SISO link between the $\mathrm{D}_{\mathrm{M}}$ and GS, which is thought of as a slanted link in which the path length changed as the slope angle $\theta$ or the altitude $h$ varying independently or together.

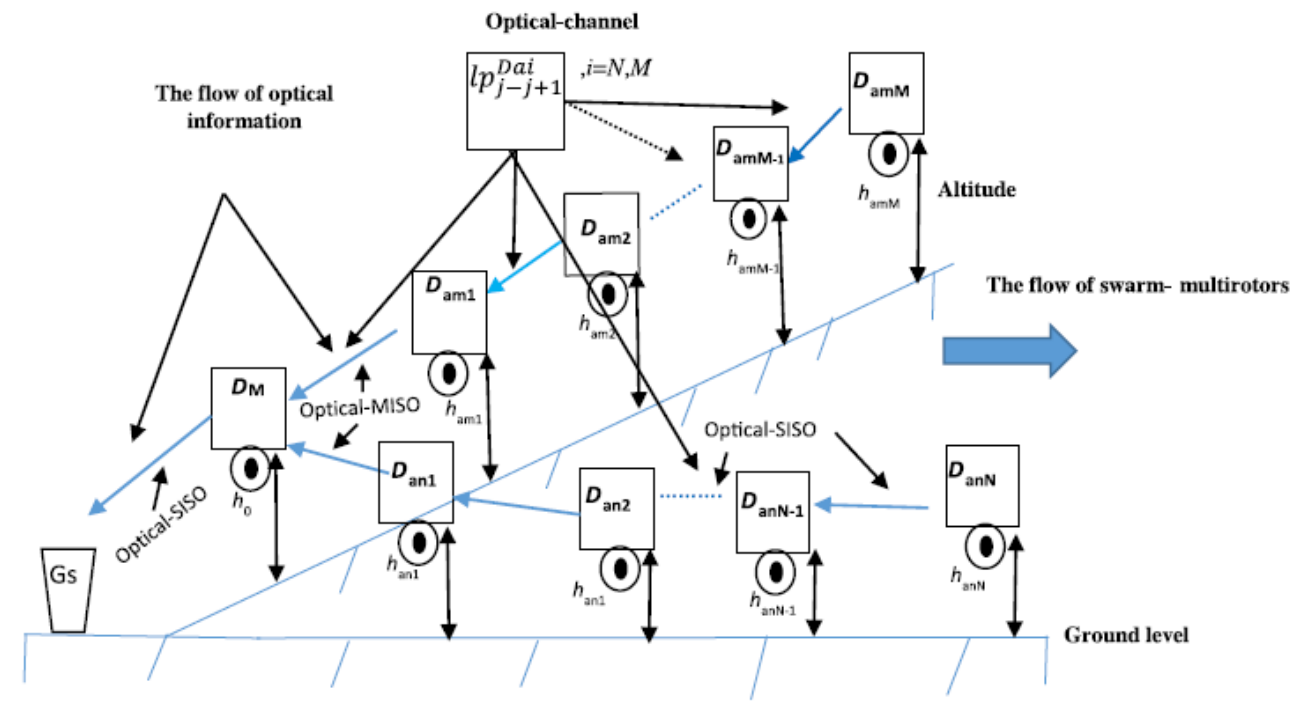

Figure 1. The V-shape swarm FSO-drones perspective illustration [13] 
There are two scenarios for the flow of the drone swarm. The first scenario adopted is when the drone swarm moves away from the GS, as shown in Figure 1, where the link between the GS and the total system will increase. As Pythagoras' theorem, these increases in the link distance $Z$ can occur due to expanding the system altitude $h$ and constant slope angle $\theta$ of the link or decreasing in the slope angle $\theta$ at fixed system altitude $h$ as clarified in Figure 2. The second scenario is when the drone swarm flow toward the GS reduces the link distance $Z$ between the GS and the flying system in two cases, reducing $Z$ due to changing the altitude $\mathrm{h}$ and the slope angle $\theta$ or changing $\theta$ at fixed $h$. The first scenario was adopted to get the distances at which the system failed at a specific pointing error angle $\theta_{r}$.

The system performance was determined using the onboard decode-and-forward (DAF) technique. The DAF technique denotes that the optical signal is transmitted from the last drone in an arm such as $\mathrm{D}_{\mathrm{aM}}$ to $\mathrm{D}_{\mathrm{aM1} 1 \mathrm{1}}$ until the signal reaches the drone $\mathrm{D}_{\mathrm{M}}$; then, $\mathrm{D}_{\mathrm{M}}$ decodes and forward to GS. This paper assumes that the drones move at a constant speed and change their altitude $h$ simultaneously, and the drones will flow on the opposite side of the GS. In this paper, the number of drones considered is five drones, and the increase is also possible, and that depends on the required application. The system parameters are as shown in Table 1. The parameters $Z$ and $\theta_{r}$ were set as varying values, and their values were inspected. The parameter $h$ values depend on the drone specification referred to in the drone datasheet [14] and depend on the required test, as shown in the results and discussion section.

Table 1 . The system parameters

\begin{tabular}{lcc}
\hline \multicolumn{1}{c}{ Parameter } & Symbol & Value \\
\hline Transmission Rate & Rate & $1 \mathrm{Gbps}$ \\
Optical Transmitted Power & $P_{t}$ & $40 \mathrm{~mW}(16 \mathrm{dBm})$ \\
Responsivity & $R$ & 1 \\
Distance between Tx and Rx & $Z$ & Varying \\
Altitude & $h$ & Varying \\
Wavelength & $\lambda$ & $1550 \times 10^{-9} \mathrm{~nm}$ \\
Receiver Diameter & $D_{R}$ & $1 \mathrm{~cm}$ to $10 \mathrm{~cm}$ \\
Pointing Error Angle & $\theta_{r}$ & Varying \\
Beam Waist at z=0 & $w_{0}$ & $5 \times 10^{-2}$ \\
\hline
\end{tabular}

\subsection{Channels modeling}

The mathematical model of each topology in the proposed system is expressed, including the channel gain $\mathrm{H}$ and its related factors and the probability density function probability density function (PDF) of the $\mathrm{H}$ in weak atmospheric turbulence using Log-normal distribution. The channel's gain factors formulas have been found in [15]. These factors were modified to suit the system's topologies in this paper.

The Log-normal distribution characterized by a scintillation index $\sigma_{\mathrm{I}}^{2}<1$. The primary source of scintillation is as a result of fluctuations (temperature variations) in the index of refraction $n$, and commonly known as optical turbulence and expressed as [16]:

$$
\sigma_{I}^{2}=\exp \left(\sigma_{R}^{2}\right)-1
$$

where $\sigma_{R}^{2}$ is the Rytov variance expressed as:

$$
\sigma_{R}^{2}=1.23 C_{n}^{2}(h) K^{7 / 6} Z^{11 / 6}
$$

where $K=2 \pi / \lambda$ is the optical wavenumber [16].

As the index of refraction $n$ fluctuated, the refractive index structure parameter $C_{n}^{2}$ that is a function of altitude $h$ and found using Hufnagel-Valley Boundary (HVB) as [17]:

$$
C_{n}^{2}(h)=0.00594\left[\begin{array}{c}
\left(\frac{V}{27}\right)^{2}\left(10^{-5} h\right)^{10} \exp (-h / 1000)+2.7 \times 10^{-16} \exp \left(-\frac{h}{1500}\right) \\
+A \exp \left(-\frac{h}{100}\right)
\end{array}\right] \mathrm{m}^{-2 / 3}
$$

where $v$ is the wind speed mean square value in $\mathrm{m} / \mathrm{s}$, and $A$ is a nominal value of $\mathrm{C}_{\mathrm{n}}^{2}(0)$ at the ground level in $\mathrm{m}^{-2 / 3}$. The $C_{n}^{2}$ value varies during daytime and at night and varies depending on the turbulence regime's strength (weak, moderate, strong, and saturation) [18].

The transmitted optical signal in a weak atmospheric regime modulated using pulse position modulation (PPM). The received optical signal $y$ is [19]:

$$
y=x R \sum_{m=1}^{M} H_{m}+v_{n} \quad m=1,2,3, \ldots . M
$$


where $M$ is the number of channels in the topology (for SISO M=1, and MISO=2), $x$ is the transmitted signal, $R$ is the photodetector responsivity, $v_{n}$ is the AWGN with variance $\sigma_{n}=N_{0} / 2$, and $H_{m}$ is the channel gain of the $\mathrm{m}^{\text {th }}$ channel. The total channel gain $H$ is given by:

$$
H=\prod_{m=1}^{M} H_{a_{m}} H_{f_{m}} H_{p_{m}} \quad m=1,2,3, \ldots M
$$

where the factors $H_{a_{m}}, H_{f_{m}}$, and $H_{p_{m}}$ denotes the attenuation due to beam extinction arising from scattering and absorption, scintillation effects, and geometric spread and pointing loss of the $\mathrm{m}^{\text {th }}$ channel, respectively. The attenuation $H_{a_{m}}$ does not exhibit randomness in its behavior; hence, perceived as a deterministic component with a relatively long interval of time on the order of hours compared to the bit duration in ranges of nanoseconds or less. On the other hand, $H_{f_{m}}$ and $H_{p_{m}}$ are time-variant factors, which demonstrate variations in the fading channel on the order of milliseconds [20]. The atmospheric attenuation $H_{a_{m}}$ has been modeled by the Beer-Lambert law expressed as [21]:

$$
H_{a_{m}}=\exp \left(-Z_{m} \sigma\right)
$$

where $\sigma$ is the attenuation coefficient, which is a function of visibility [22], and $Z_{m}$ is the path length of the $\mathrm{m}^{\text {th }}$ channel. The atmospheric turbulence $H_{f_{m}}$ is the channel fading can be expressed as:

$$
H_{f_{m}}=\frac{2 P_{r_{m}}}{\pi W_{m}}
$$

where $P_{r_{m}}$ and $W_{\mathrm{m}}$ are the received optical power and the beam spot radius of the $\mathrm{m}^{\text {th }}$ channel at the receiver, respectively. The pointing error $H_{p_{m}}$ of the optical beam can be expressed as:

$$
H_{p_{m}}=A_{0_{m}} \exp \left(\frac{-2 r_{m}^{2}}{W_{Z e q_{m}}^{2}}\right)
$$

where $r_{m}=Z . \theta_{r_{m}}$ is the radial displacement of the optical beam that is between the aperture center and the beam center, as shown in Figure 2, where $\theta_{r_{m}}$ is pointing error angle (divergence angle) of the $\mathrm{m}^{\text {th }}$ channel that must be $<10^{-4} \mathrm{rad}$ [23], where this value is for long link distance. For a short distance, $\theta_{r_{m}}$ can be set $>$ $10^{-4} \mathrm{rad}$ as proved in the results section, $A_{0_{m}}=\left[\operatorname{erf}\left(v_{m}\right)\right]^{2}$ is the fraction of collected power at the radial displacement $r=0$ (no pointing error occurs), and $W_{Z e q_{m}}$ is the equivalent beam radius of the optical beam in the $\mathrm{m}^{\text {th }}$ channel that can express as [15]:

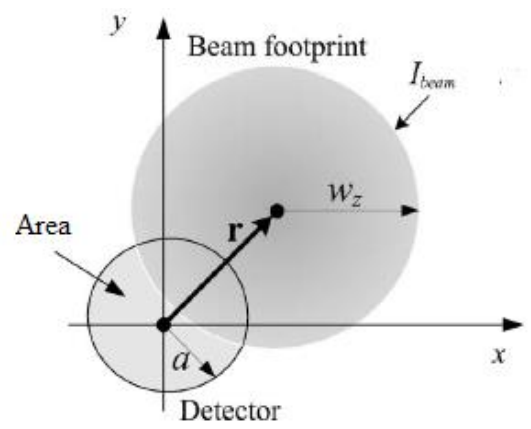

Figure 2. Detector and beam footprint with misalignment on the detector plane [24]

$$
W_{Z e q_{m}}=\mathrm{w}_{Z_{m}}^{2} \frac{\sqrt{\pi} \operatorname{erf}\left(v_{m}\right)}{2 \mathrm{v} \exp \left(-v_{m}^{2}\right)}
$$

where $w_{z_{m}}$ is the beam radius at which the intensity drops to $e^{-2}$ of the axial value at a distance $Z_{m}$ and is related to the beam waist $w_{0}$ at $Z_{m}=0$ as $w_{z_{m}} \approx w_{0}\left[1+\varepsilon_{m}\left(\frac{\lambda z_{m}}{\pi w_{0}^{2}}\right)^{2}\right]^{\frac{1}{2}}$, where $\varepsilon_{m}=\left(1+2 w_{0}^{2} / \rho_{0}^{2}\left(Z_{m}\right)\right)$, and the coherence length $\rho_{0}\left(Z_{m}\right)=\left(0.55 C_{n}^{2}(h) K^{2} Z\right)^{-3 / 5}$, and $v_{m}=(\sqrt{\pi} a) /\left(\sqrt{2} w_{z_{m}}\right)$, where $a$ is the receiver radius [24]. The validation of the Gaussian beam model requires $w_{0}>2 \lambda / \pi$ [23]. The PDF of the channel gain $H$ for the SISO topology in weak turbulence using Log-normal distribution has been expressed as [24]: 


$$
f_{h}(H ; w Z)=\frac{\gamma^{2}}{2\left(A_{0} H_{a}\right)^{2}} H^{\gamma^{2}-1} \times \operatorname{erfc}\left(\frac{\ln \left(\frac{H}{A_{0} H a}\right)+\mu}{\sqrt{8} \sigma_{\chi}}\right) e^{\left(2 \sigma_{\chi}^{2} \gamma^{2}\left(1+\gamma^{2}\right)\right)}
$$

The PDF of the channel gain $H_{m}$ for the $m^{\text {th }}$ channel in MISO topology is similar to the PDF of SISO topology with the difference of dividing the variance of the log-normal channel by the number of transmitting antennas [25] and can express as:

$$
f_{H_{m}}\left(H_{m} ; w_{z_{m}}\right)=\frac{\gamma_{m}^{2}}{2\left(\mathrm{~A}_{0}^{\mathrm{m}} H_{a_{m}}\right)^{\gamma^{2}}} H_{m}^{\gamma_{m}^{2}-1} \times \operatorname{erfc}\left(\frac{\ln \left(\frac{H_{m}}{\mathrm{~A}_{0}^{\mathrm{m}} H_{m}}\right)+\mu_{m}}{\sqrt{2} \sigma_{x_{m}}}\right) e^{\left(\sigma_{x_{m}}^{2} \gamma_{m}^{2}\left(1+\gamma_{m}^{2}\right)\right)}
$$

then the full PDF of the MISO topology is given as:

$$
f_{M I S O}^{T}=\sum_{m=1}^{M} f_{H_{m}}\left(H_{m} ; w_{z_{m}}\right) \quad m=1,2,3, \ldots . M
$$

The parameters in (10) and (11) defined as: $\gamma_{m}=\frac{W_{Z e q_{m}}}{2 \sigma_{S}}$ is the ratio between the equivalent beam radius at the receiver and the pointing error displacement standard deviation at the receiver, $\mu_{m}=\sigma_{x_{m}}^{2}\left(1+2 \gamma_{m}^{2}\right)$, and $\sigma_{x_{m}}^{2}$ is the log-amplitude variance of the MISO channel expressed for plane wave as:

$$
\sigma_{x_{m}}=0.30545 K^{7 / 6} C_{n}^{2}(h) Z_{m}^{11 / 6} \approx \frac{\sigma_{R m}}{4}
$$

The SISO channel $m=1$ ( just one channel). For Simplicity, the two channels of the MISO topology have been assumed equal where the parameters of the two SISO arms were assumed equal.

\subsection{The average bit error rate (ABER)}

The ABER is the instantaneous conditional BER(H) product by the PDF of the channel gain $H$ [6]. For each channel in the system, the ABER was measured and given by:

$$
A B E R=\int_{0}^{\infty} B E R(H) \times f_{H}(H) d H
$$

\subsubsection{ABER of the SISO channels}

For the SISO channels, the solution of the integration of the PDF of (10) as given in [24]:

$$
f_{H}\left(H ; w_{z}\right)=\frac{1}{2}\left[\exp ^{\gamma^{2} \Psi-2 \sigma_{x}^{2} \gamma^{4}} \operatorname{erfc}\left(\frac{\psi}{\sqrt{8} \sigma_{x}}\right)+\operatorname{erfc}\left(\frac{4 \sigma_{x}^{2} \gamma^{2}-\Psi}{\sqrt{8} \sigma_{x}}\right)\right]
$$

where $\Psi=\ln \left(\frac{h_{0}}{A_{0} H_{a}}\right)+u$, and $h_{0}=\sqrt{C^{-1}\left(R_{0}\right) \sigma_{n}^{2} / 2 P_{t}^{2} R^{2}}$, where $C$ is the channel capacity that is the maximum achievable data rate that reliably communicated between the transmitter and receiver, $R_{0}$ is the data rate, $\sigma_{n}^{2}$ is noise variance, $P_{t}$ is the transmitted power, and $R$ is the photodetector responsivity [24]. Substitute (15) into (14), the ABER becomes:

$$
A B E R=B E R_{\operatorname{sim}}(H) \times \frac{1}{2}\left[\exp ^{\gamma^{2} \Psi-2 \sigma_{x}^{2} \gamma^{4}} \operatorname{erfc}\left(\frac{\Psi}{\sqrt{8} \sigma_{x}}\right)+\operatorname{erfc}\left(\frac{4 \sigma_{x}^{2} \gamma^{2}-\Psi}{\sqrt{8} \sigma_{x}}\right)\right]
$$

\subsubsection{ABER of the MISO channel}

For the MISO channel, the integration solution of (11) of the PDF of the $\mathrm{m}^{\text {th }}$ channel gain $H$ is given by:

$$
f_{H_{m}}\left(H_{m} ; w_{z_{m}}\right)=\frac{1}{2}\left[\exp ^{\gamma_{m}^{2} \Psi_{m}-\sigma_{x_{m}}^{2} \gamma_{m}^{4}} \operatorname{erfc}\left(\frac{\Psi_{m}}{\sqrt{2} \sigma_{x_{m}}}\right)+\operatorname{erfc}\left(\frac{2 \sigma_{x_{m}}^{2} \gamma_{m}^{2}-\Psi_{m}}{\sqrt{2} \sigma_{x_{m}}^{2}}\right)\right]
$$

where $\Psi_{m}=\ln \left(\frac{h_{0}}{A_{0_{m}} H_{a_{m}}}\right)+\mu_{m}$, substituted (18) into (15) yields:

$$
A B E R_{M I S O}^{m}=B E R_{\text {sim }}\left(H_{m}\right) \times \frac{1}{2}\left[\exp ^{\gamma_{m}^{2} \Psi_{m}-\sigma_{x_{m}}^{2} \gamma_{m}^{4}} \operatorname{erfc}\left(\frac{\Psi_{m}}{\sqrt{2} \sigma_{x_{m}}}\right)+\operatorname{erfc}\left(\frac{2 \sigma_{x_{m}}^{2} \gamma_{m}^{2}-\Psi_{m}}{\sqrt{2} \sigma_{x_{m}}^{2}}\right)\right]
$$

Then, the ABER of the MISO topology is given by:

$$
A B E R_{M I S O}=1-\prod_{m=1}^{M}\left(1-A B E R_{M I S O}^{m}\right) \quad m=1,2,3, \ldots . M
$$


The total ABER for the overall system calculated as [13]:

$$
T A B E R=1-\prod_{i=1}^{N}\left(1-A B E R_{i}\right) \quad i=1,2,3, \ldots . N
$$

where $i$ is the $i^{\text {th }}$ drone, $N$ is the number of drones that equal the number of channels in the proposed system, and $A B E R_{i}$ is the average bit error rate for each channel.

\section{THE RESULTS AND DISCUSSION}

The results got from the expressions mentioned in the previous sections. The results showed that the system performance in terms of TABER $\approx 10^{-8}$. This paper's central axis is to determine the threshold values of the pointing error angles $\theta_{r}$ at particular link distance $Z$ where the system keeps its high performance and extract the values at which the system failed. The recommended pointing angle $\theta_{r}<10^{-4} \mathrm{rad}$ for long-distance application (inter-satellite laser communication), where the receiver diameter $D_{R}$ fixed to $250 \mathrm{~cm}[23]$.

First of all, test the pointing error angles $\theta_{r}=10^{-3}, 10^{-4}$, and $10^{-5} \mathrm{rad}$ at receiver diameter $D_{R}=1 \mathrm{~cm}$. The results as shown in Figure 3 demonstrated that at link distance $Z=1050 \mathrm{~m}$, the pointing error angle $\theta_{r}=10^{-3}$ rad was failed and could not apply it beyond $Z>1050 \mathrm{~m}$. The altitude in this test can be set to $h \leq 1050 \mathrm{~m}$ (when $h=Z$, the swarm is perpendicular to the GS).

The second test was to see the effect of increasing the receiver diameter $D_{R}$ to be $10 \mathrm{~cm}$ at the same parameters. The result shown in Figure 4 demonstrated that the system did not fail and got high performance for $\theta_{r}=10^{-3}$ rad because the receiver captures optical signals more.

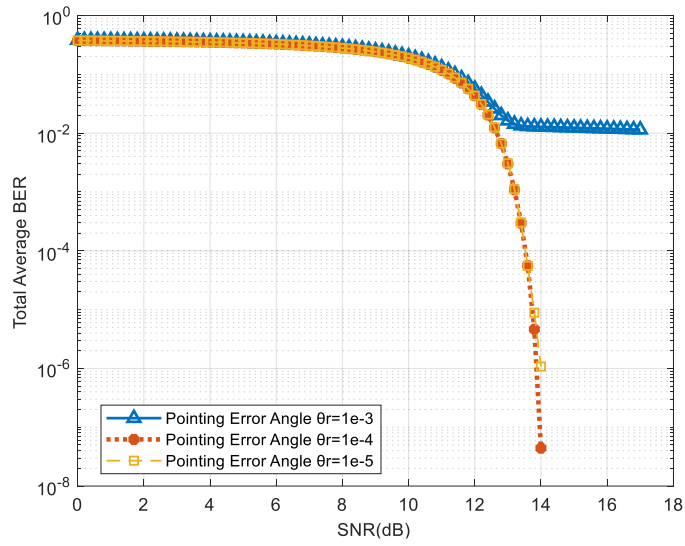

Figure 3. The system failed at $Z=1050 \mathrm{~m}$ and $D_{R}=1 \mathrm{~cm}$

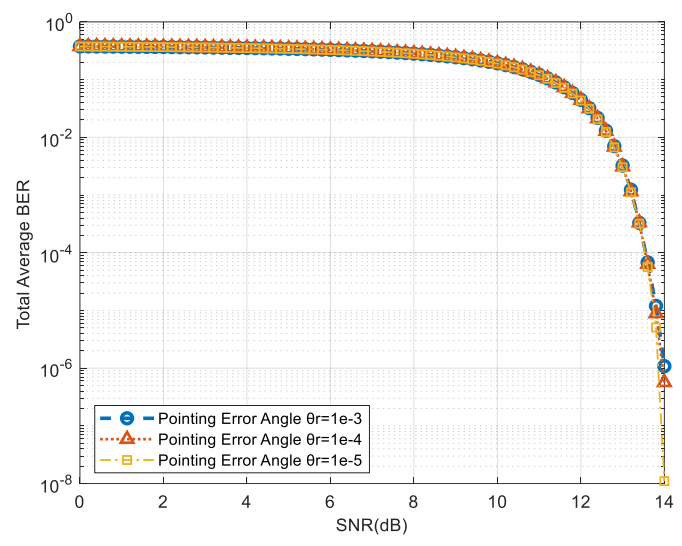

Figure 4. The system performance at $Z=1050 \mathrm{~m}$ and $D_{R}=10 \mathrm{~cm}$

Now, test the pointing error angles $\theta_{r}=10^{-4}$ and $10^{-5}$ rad to see at which link distance $Z$ the drone swarm system can be applied. The result shown in Figure 5 demonstrated that the link distance $Z \leq 8500 \mathrm{~m}$ can the swarm reach at the receiver diameter $D_{R}=10 \mathrm{~cm}$. The value of the altitude $h$ can be set to $h \leq 8500 \mathrm{~m}$ also.

After examining the link distance $Z$ for the pointing error angle $\theta_{r}=10^{-3}$ rad at receiver diameter $D_{R}=1 \mathrm{~cm}$, further examining the link distances range for $\theta_{r}=10^{-3}$ rad that can be applied when increasing the receiver diameter by one centimeter each time. The result shown in Figure 6 is when the $D_{R}=2 \mathrm{~cm}$ and demonstrated that the system got a high performance at $Z \leq 1059 \mathrm{~m}$, and the system failed at $Z=1060 \mathrm{~m}$. Table 2 summarized the results of the increase in the receiver diameter $D_{R}$ from $1 \mathrm{~cm}$ to $10 \mathrm{~cm}$ results in an additional range of link distances $Z$ for the pointing error angles $\theta_{r}=10^{-3} \mathrm{rad}$.

The increase in the receiver diameter $D_{R}$ corresponds to an increase in the received optical power, and this means the receiver capture a high percentage of the optical beam as a result of an increase in the link distances $Z$ and the beamwidth $\left(2 \times W_{z}\right)$ for the same pointing error angle $\theta_{r}=10^{-3} \mathrm{rad}$. When $D_{R} \leq 5 \mathrm{~cm}$, the increase in the permitted link distance is $<50 \mathrm{~m}$. When $D_{R} \geq 6 \mathrm{~cm}$, the increase in the link distance $\mathrm{Z}$ is $\geq 100 \mathrm{~m}$. 
Further, test the link distances $Z$ range when increasing the pointing error angle $\theta_{r}$ to $10^{-2}$ rad and the receiver diameter $D_{R}$ increases by one centimeter each time. Figure 7 demonstrated that the system's high performance was at $Z \leq 96 \mathrm{~m}$, and the system failed at $Z=97 \mathrm{~m}$ for the receiver diameter $D_{R}=1 \mathrm{~cm}$.

The increases in the receiver diameter $D_{R}$ to $2 \mathrm{~cm}$ correspond to increases in the range of link distances $Z$ at the same pointing error $\theta_{r}=10^{-2}$. As shown in Figure 8, the system's high performance at $Z \leq 99 \mathrm{~m}$ and the system failed at $Z=100 \mathrm{~m}$.

Table 3 summarized the results of the increase in the receiver diameter $D_{R}$ from $1 \mathrm{~cm}$ to $10 \mathrm{~cm}$ results in an additional range of link distances for the pointing error angles $\theta_{r}=10^{-2} \mathrm{rad}$. At $\theta_{r}=10^{-2} \mathrm{rad}$, the increase in receiver diameter $D_{R}$ results in a few increases in the link distance $Z$, mainly when $D_{R} \leq 5 \mathrm{~cm}$ corresponds to an increase in $Z \leq 5 \mathrm{~m}$. When $D_{R}>5 \mathrm{~cm}$, the increase in $Z \geq 10 \mathrm{~m}$.

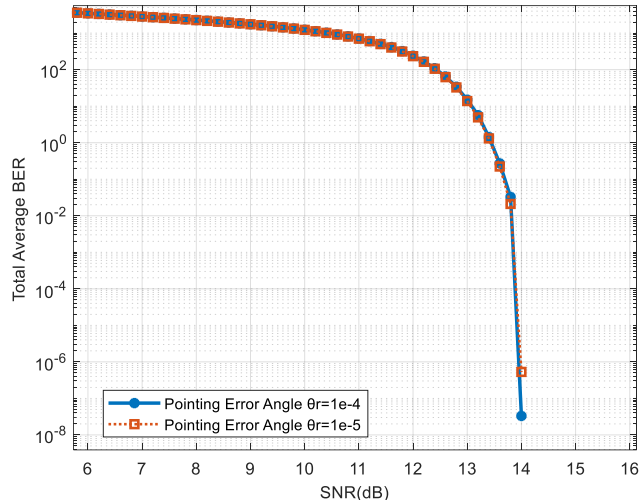

Figure 5. The system performance for $\theta_{r}=10^{-4}$ and $10^{-5} \mathrm{rad}$ at $Z=8500 \mathrm{~m}$ and $D_{R}=10 \mathrm{~cm}$

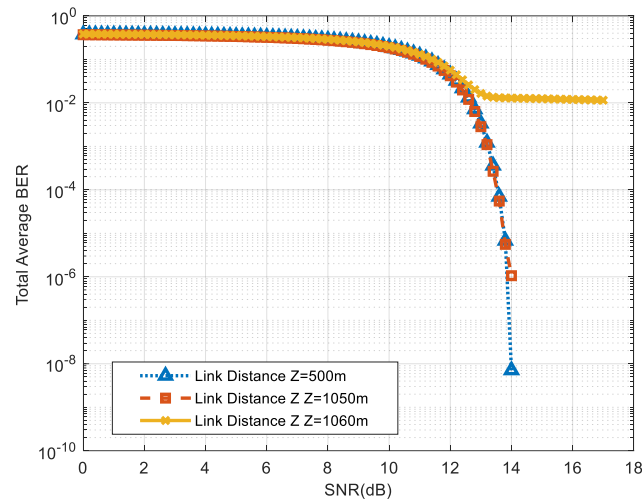

Figure 6. The system failed at $\theta_{r}=10^{-3} \mathrm{rad}, \mathrm{Z}=1060$ $\mathrm{m}$, and $D_{R}=2 \mathrm{~cm}$

Table 2. The range of link distances at $\theta_{r}=10^{-3} \mathrm{rad}$

\begin{tabular}{ccccc}
\hline $\begin{array}{c}\text { The Receiver } \\
\text { Diameter } \mathrm{D}_{\mathrm{R}}(\mathrm{cm})\end{array}$ & $\begin{array}{c}\text { The applicable } \\
\text { distance } Z(\mathrm{~m})\end{array}$ & $\begin{array}{c}\text { The system } \\
\text { failed }(\mathrm{m})\end{array}$ & $\begin{array}{c}\text { The system } \\
\text { altitude } h(\mathrm{~m})\end{array}$ & $\begin{array}{c}\text { Beam Waist } \\
W z(\mathrm{~cm})\end{array}$ \\
\hline 1 & $1 \sim 1049$ & 1050 & $1 \sim<1050$ & 5.44 \\
2 & $1 \sim 1059$ & 1060 & $1 \sim<1060$ & 5.45 \\
3 & $1 \sim 1086$ & 1087 & $1 \sim<1087$ & 5.47 \\
4 & $1 \sim 1129$ & 1130 & $1 \sim<1130$ & 5.51 \\
5 & $1 \sim 1183$ & 1184 & $1 \sim<1184$ & 5.56 \\
6 & $1 \sim 1254$ & 1255 & $1 \sim<1255$ & 5.63 \\
7 & $1 \sim 1341$ & 1342 & $1 \sim<1342$ & 5.72 \\
8 & $1 \sim 1444$ & 1445 & $1 \sim<1445$ & 5.85 \\
9 & $1 \sim 1565$ & 1566 & $1 \sim<1566$ & 6 \\
10 & $1 \sim 1702$ & 1703 & $1 \sim<1703$ & 6.19 \\
\hline
\end{tabular}

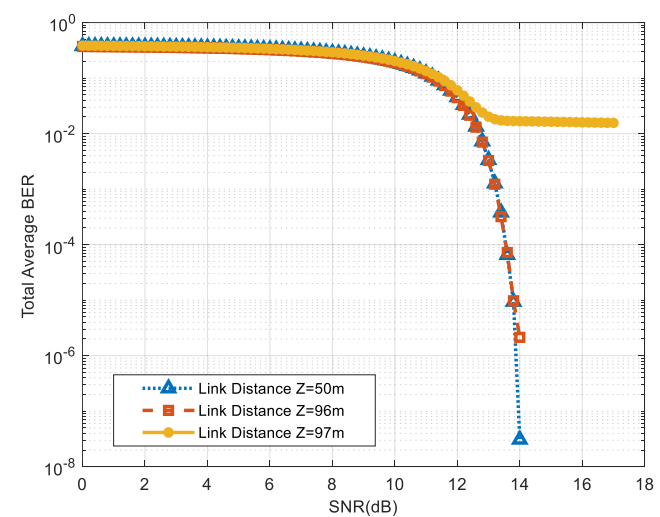

Figure 7. The system failed at $\theta_{r}=10^{-2} \mathrm{rad}$, $Z=96 \mathrm{~m}$, and $D_{R}=1 \mathrm{~cm}$

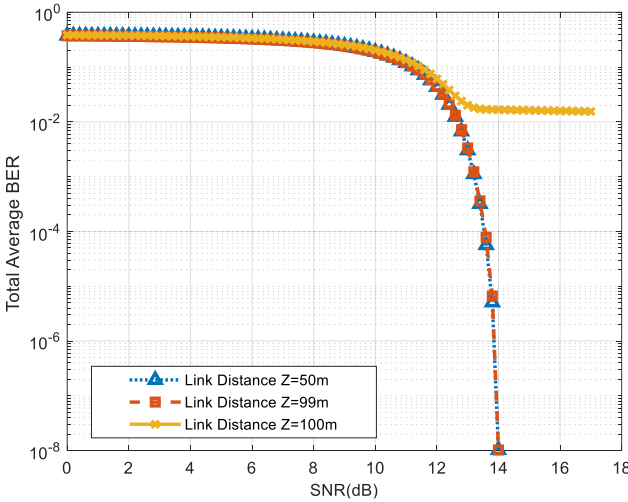

Figure 8. The system failed at $\theta_{r}=10^{-2} \mathrm{rad}$, $\mathrm{Z}=100 \mathrm{~m}$, and $D_{R}=2 \mathrm{~cm}$ 


\begin{tabular}{ccccc}
\multicolumn{5}{c}{ Table 3. The range of link distances at $\theta_{r}=10^{-2}$ rad } \\
\hline $\begin{array}{c}\text { The Receiver } \\
\text { Diameter } D_{\mathrm{R}}(\mathrm{cm})\end{array}$ & $\begin{array}{c}\text { The applicable } \\
\text { distance } Z(\mathrm{~m})\end{array}$ & $\begin{array}{c}\text { The system } \\
\text { failed }(\mathrm{m})\end{array}$ & $\begin{array}{c}\text { The system } \\
\text { altitude } h(\mathrm{~m})\end{array}$ & $\begin{array}{c}\text { Beam Waist } \\
\mathrm{Wz}(\mathrm{cm})\end{array}$ \\
\hline 1 & $1 \sim 96$ & 97 & $1 \sim<97$ & 5 \\
2 & $1 \sim 99$ & 100 & $1 \sim<100$ & 5 \\
3 & $1 \sim 100$ & 101 & $1 \sim<101$ & 5 \\
4 & $1 \sim 104$ & 105 & $1 \sim<105$ & 5 \\
5 & $1 \sim 109$ & 110 & $1 \sim<110$ & 5 \\
6 & $1 \sim 116$ & 117 & $1 \sim<117$ & 5.01 \\
7 & $1 \sim 126$ & 127 & $1 \sim<127$ & 5.01 \\
8 & $1 \sim 136$ & 137 & $1 \sim<1445$ & 5.01 \\
9 & $1 \sim 151$ & 152 & $1 \sim<152$ & 5.01 \\
10 & $1 \sim 169$ & 170 & $1 \sim<170$ & 5.01 \\
\hline
\end{tabular}

\section{CONCLUSION}

The simulated V-shape system consists of two topologies: SISO and MISO topology, and each topology can be considered an independent subsystem. The closed-form expression of the ABER for the two topologies and the total ABER for the whole system was derived and determined. The three factors of the channel gain $H$ were considered. The pointing error $H_{p}$ factor parameters are the pointing error angle $\theta_{r}$ and link distance $Z$. We manipulated its values to extract the permitted and applicable values that preserve the system at high performance. The increase in the pointing error angle $\theta_{r}$ is not recommended and means that the line-of-sight (LOS) between the transmitter and receiver is not precise.

All the results showed that at SNR $<10 \mathrm{~dB}$ there is a trivial betterment in the ABER. After $\mathrm{SNR} \geq 10 \mathrm{~dB}$, there are noticeable improvements in system performance. Also, the results showed that further increase the pointing error angle $\theta_{r}$ (become worst; consequently, the performance becomes worst) must correspond decrease in path length $Z$ to get high performance. The increase in the link distance makes the beam broader, and the receiver catches part of the incident optical; therefore, the radial displacement $r$ $\left(r \propto \theta_{r}\right)$ must be as small as possible. The results enable the GS to monitor and control the system performance by changing the link distances $\mathrm{Z}$ depending on the measured performance. This paper can be considered as a benchmark in the optical communication field. For future work, optimization for the crucial parameters such as the transmitted power and the receiver aperture diameter affected the system performance.

\section{REFERENCES}

[1] I. S. Ansari, F. Yilmaz, and M. S. Alouini, "Impact of pointing errors on the performance of mixed RF/FSO dual-hop transmission systems," IEEE Wirel. Commun. Lett., vol. 2, no. 3, pp. 351-354, June 2013, doi: 10.1109/WCL.2013.042313.130138.

[2] Y. Jiao, J. Wang, X. Dang, M. Chen, W. Hu, and Y. Huang "Performance Analysis of Multihop Free Space Optical Communication System with Pointing Errors," 9th International Conference on Optical Communications and Networks (ICOCN 2010), 2010, pp. 290-293, doi: 10.1049/cp.2010.1208.

[3] G. Immadi, M. Venkata Narayana, A. Sree Madhuri, and V. L. Tejaswani Sabbasani, "Simulation Of Free Space Optical Communication Under Different Weather Conditions," International Journal of Pure and Applied Mathematics, vol. 117, no. 18, pp. 143-148, 2017.

[4] W. Fawaz, C. Abou-rjeily, and C. Assi, "UAV-Aided Cooperation for FSO Communication Systems," IEEE Communications Magazine, vol. 56, no. 1, pp. 70-75, Jan. 2018, doi: 10.1109/MCOM.2017.1700320.

[5] E. Leitgeb, K. Zettl, S. S. Muhammad, N. Schmitt, and W. Rehm, "Investigation in free space optical communication links between unmanned aerial vehicles (UAVs)," Proc. 2007 9th Int. Conf. Transparent Opt. Networks, Ict. 2007, 2007, doi: 10.1109/ICTON.2007.4296268.

[6] H. G. Sandalidis, T. A. Tsiftsis, G. K. Karagiannidis, and M. Uysal, "BER Performance of FSO Links over Strong Atmospheric Turbulence Channels with Pointing Errors," IEEE Communications Letters, vol. 12, no. 1, pp. 44-46, Jan. 2008, doi: 10.1109/LCOMM.2008.071408.

[7] S. S. Muhammad, et al., "Challenges in Establishing Free Space Optical Communications Between Flying Vehicles,” 2008 6th Int. Symp. Commun. Syst. Networks Digit. Signal Process., Jul. 2008, pp. 82-86, doi: 10.1109/CSNDSP.2008.4610721.

[8] A. Hatziefremidis, K. E. Zarganis, H. C. Leligou, and N. Pleros, "Bit error rate analysis along a slanted path link between UAVs and Ground Stations," Int. Conf. Transparent Opt. Networks, Jun. 2013, pp. 1-4, doi: 10.1109/ICTON.2013.6602799.

[9] M. T. Dabiri, and S. M. S. Sadough, "Optimal Placement of UAV-Assisted Free-Space Optical Communication Systems With DF Relaying,” IEEE Communications Letters, vol. 24, no. 1, pp. 155-158, Jan. 2020, doi: 10.1109/LCOMM.2019.2949274.

[10] C. Chlestil, E. Leitgeb, N. P. Schmitt, S. S. Muhammad, K. Zettl, and W. Rehm, "Reliable optical wireless links within UAV swarms," 2006 Int. Conf. Transparent Opt. Networks, 2006, pp. 39-42, doi: 10.1109/ICTON.2006.248491. 
[11] C. Chlestil, et al., "Optical Wireless on Swarm UAVs for High Bit Rate Applications," Proc. IEEE Conf. CSNDSP, 2006, pp. 634-638.

[12] C. R. Shah, "Performance and Comparative Analysis of SISO, SIMO, MISO, MIMO," International Journal of Wireless Communication and Simulation, vol. 9, no. 1, pp. 1-14, 2017.

[13] W. J. Mazher, H. T. Ibrahim, O. N. Ucan, and O. Bayat, "Drone swarm with free-space optical communication to detect and make deep decisions about physical problems for area surveillance," Optical Engineering, vol. 57, no. 3, March 2018, doi: 10.1117/1.OE.57.3.036116.

[14] SZ DJI Technology Co. Ltd., "Matrice 600," 2017. [Online]. Available: https://www.dji.com/matrice600

[15] Md. T. Rahman, S. Iqbal, and Md. M. Islam "Modeling and Performance Analysis of Free Space Optical Communication System," 2012 International Conference on Informatics, Electronics and Vision, ICIEV 2012, no. April 2019, 2012, doi: 10.1109/ICIEV.2012.6317371.

[16] M. R. Abaza, N. A. Mohammed, M. H. Aly, "BER Performance of M -ary PPM Free-Space Optical Communications with Channel Fading," 8th International Conference on High-Capacity Optical Networks and Emerging Technologies, HONET 2011, no. December, 2011, pp. 2-7, doi: 10.1109/HONET.2011.6149799.

[17] H. Kaushal and G. Kaddoum, "Optical Communication in Space : Challenges and Mitigation Techniques," IEEE Commun. Surv. Tutorials, vol. 19, no. 1, pp. 57-96, 2017, doi: 10.1109/COMST.2016.2603518.

[18] S. M. Navidpour, M. Uysal, and M. Kavehrad, "BER Performance of Free-Space Optical Transmission with Spatial Diversity," IEEE Transactions on Wireless Communications, vol. 6, no. 8, pp. 2813-2819, 2007, doi: 10.1109/TWC.2007.06109.

[19] K. Prabu, D. S. Kumar, and R. Malekian, "BER analysis of BPSK-SIM-based SISO and MIMO FSO systems in strong turbulence with pointing errors," Opt. - Int. J. Light Electron Opt., vol. 125, no. 21, pp. 6413-6417, 2014, doi: 10.1016/j.ijleo.2014.08.006.

[20] I. E. Lee, Z. Ghassemlooy, W. P. Ng, and M. A. Khalighi, "Reducing Pointing Errors in Free-Space Optical Communication Links over Turbulences with a Partially Coherent Gaussian Beam," 2016 IEEE Int. Conf. Commun. Work., 2016, pp. 163-168, doi: 10.1109/ICCW.2016.7503782.

[21] N. A. Mohammed, A. S. El-wakeel, and M. H. Aly, "Pointing Error in FSO Link under Different Weather Conditions," Int. Journal of Video \& Image Processing and Network Security, vol. 12, no. 1, pp. 6-9, 2012.

[22] M. T. Dabiri, S. M. S. Sadough, and M. A. Khalighi, "Channel Modeling and Parameter Optimization for Hovering UAV-Based Free Space Optical Links," EEE Journal on Selected Areas in Communications, vol. 36, no. 9, pp. 2104-2113, 2018, doi: 10.1109/JSAC.2018.2864416.

[23] T. Song, Q. Wang, and M. Wu, "Impact of Pointing Errors on the Error Performance of Intersatellite Laser Communications," Journal of Lightwave Technology, vol. 35, no. 14, May 2017, doi: 10.1109/JLT.2017.2705132.

[24] A. A. Farid and S. Hranilovic, "Outage capacity optimization for free-space optical links with pointing errors," Journal of Lightwave Technology, vol. 25, no. 7, pp. 1702-1710, 2007, doi: 10.1109/JLT.2007.899174.

[25] M. Abaza, R. Mesleh, A. Mansour, and A. Alfalou, "MIMO Techniques for High Data Rate Free Space Optical Communication System in Log-Normal Channel," The International Conference on Technological Advances in Electrical, Electronics and Computer Engineering, TAEECE 2013, 2013, pp. 1-5, doi: 10.1109/TAEECE.2013.6557185. 